\title{
High Resolution Electron Microscopy of Carbonaceous Materials
}

\author{
Sumio Iijima
}

Department of Materials Science \& Engineering, Meijo University (also AIST \& NEC)

1-501, Shiogamaguchi, Tenpaku, Nagoya, Aichi 468-8502, Japan

In 1991, the carbon nanotubes (CNTs) were discovered. Its discovery has never been realized without HRTEM. It is of interest to mention that the discoverer, present author, reported the first HRTEM images of oxides showing atomic details in 1971 (Fig. 1) [2]. It was the next year when I joined the late Prof. J. M. Cowley group as a post-doctoral researcher at Arizona State University. We had just opened up a new era of phase contrast. During the 1970s the author experienced some HTREM observations of carbonaceous materials such as amorphous carbon [3], nano-scale small graphite balls (Fig. 2) [4], atomic sized pits on thin graphite film surfaces (Fig. 3) [5], etc. The latter one will be discussed in the latest studies here. All of these HRTEM studies have been the basis of the discovery of the CNTs. Another important study of HRTEM on the ultra-fine particles (in these days they are called nano-particles) should be mentioned, which I have worked on after returning to Japan, and developed the way of dynamic observation of nano-particles [6].

There are several methods to characterize the atomic structures of CNTs; X-ray diffraction, Raman spectroscopy, Photo luminescence spectroscopy, Scanning probe microscopy, HRTEM, Electron diffraction. Among those methods HRTEM has become the most reliable one for analyzing individual nanotubes that were described by chiral indices, which define uniquely a nanotube structure. We have developed two ways to determine the indices in HRTEM [7] and ED [8].

The most recent development in the structural analysis is of dynamic visualization of individual vacancies and interstitials occurring in graphene layers during electron beam irradiation. The results have brought an insight of understanding a damage formation of graphite by high-energy irradiation $[9,10]$.

References

[1] S. Iijima, Nature 345 (1991) 56.

[2] S. Iijima, J.Appl.Phys., 42 (1971) 5891.

[3] Sumio Iijima, J. Microscopy, 119 (1980) 99.

[4] Sumio Iijima, J. Cryst. Growth, 50 (1980) 675.

[5] Sumio Iijima, Optik, 47 (1977) 437.

[6] S. Iijima and T. Ichihashi, Phys. Rev. Lett. 56 (1986) 616.

[7] M. Kociak, K. Hirahara, K. Suenaga and S. Iijima, European Physical Journal B, 32 (2002) 457.

[8] K. Hirahara, M. Kociak, S. Bandow, H. Kataura, and S. Iijima, Phys. Rev. B 70 (2004) 205422.

[9] A. Hashimoto, K. Suenaga, A. Gloter, K. Urita, S. Iijima, Nature, 430 (2004) 870.

[10] K. Urita, K. Suenaga, T. Sugai, H. Shinohara and S. Iijima, Nano Letters (2005) accepted. 

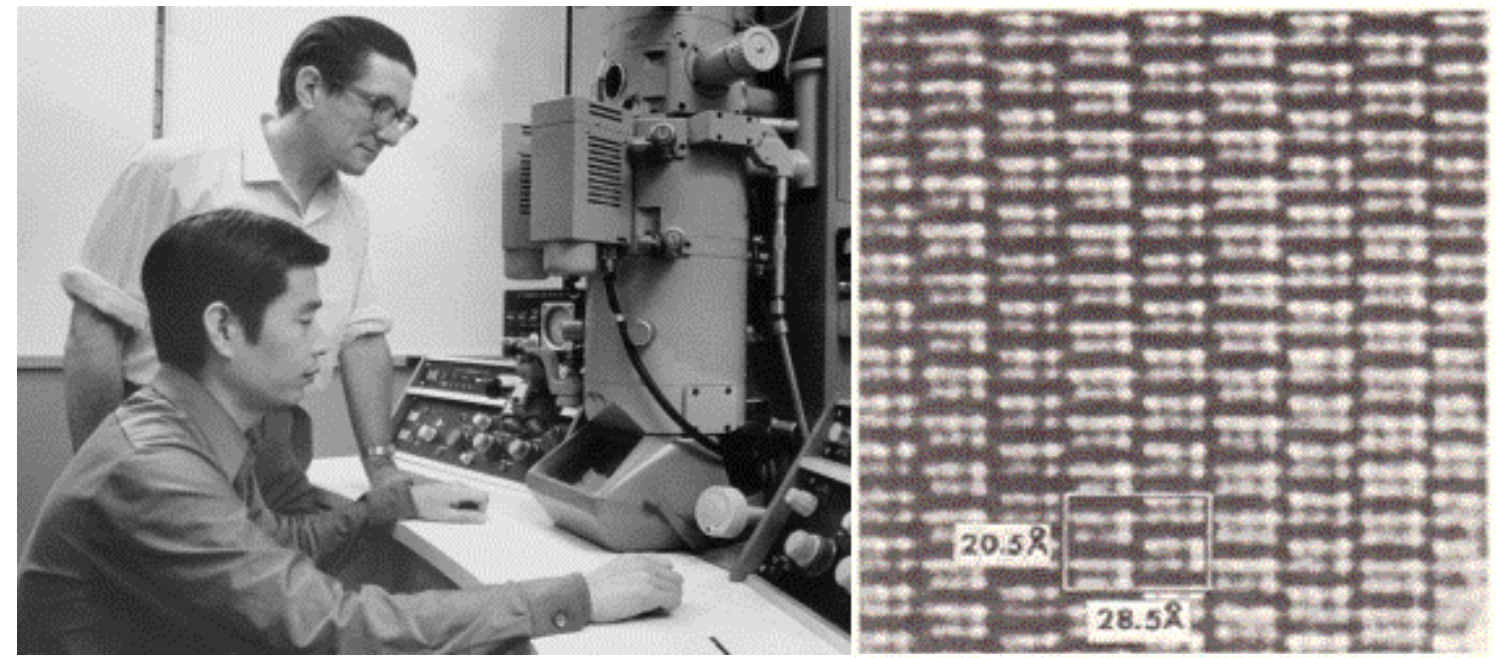

Fig.1. Professor J.M. Cowley and the author in 1976 and a HRTEM image of Nb-Ti Oxides
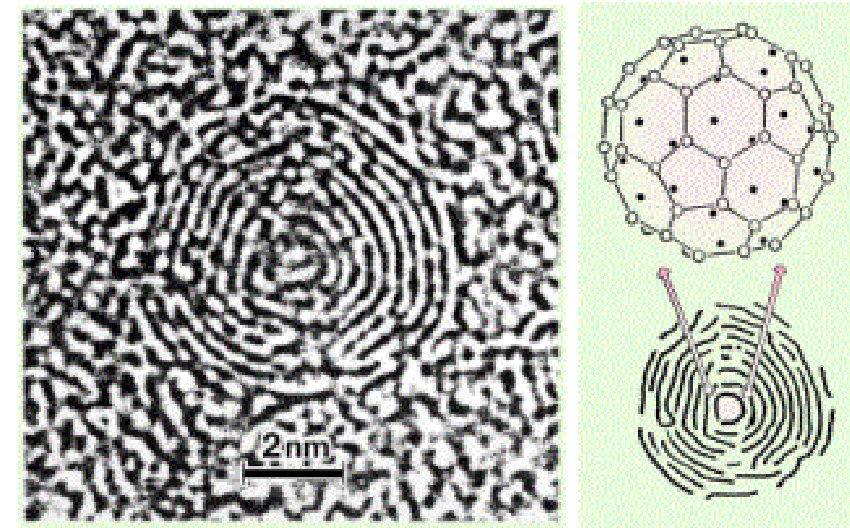

Fig. 2. Ultra-fine spherical graphite ball composed of nesting graphite shells containing a fullerene molecule, C60, at the center and its illustration.

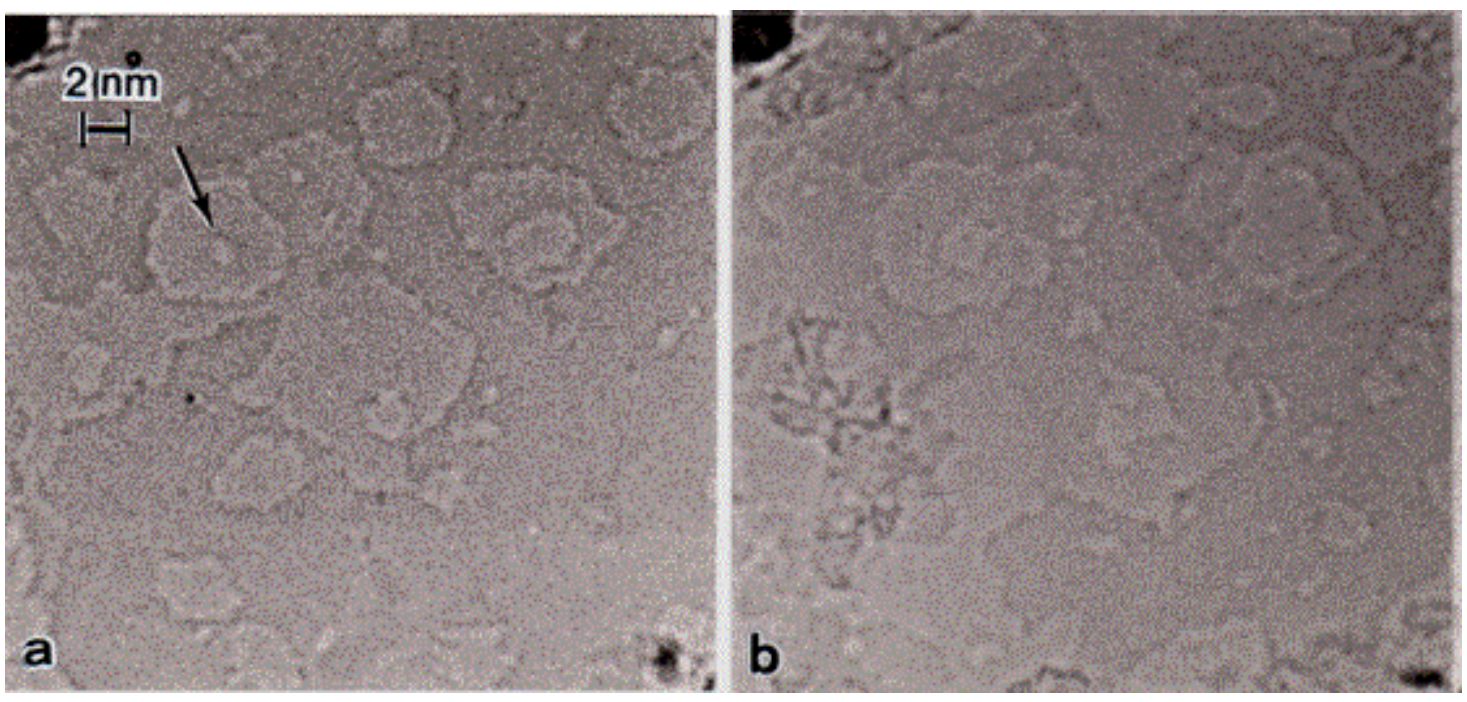

Fig. 3. HRTEM images of pit-formation with atomic high steps on the surface of this graphite. Small pits such as indicated by an arrow associated with pits of several carbon atoms vacancies. 